Case Report

\title{
An Unusual Case of Systemic Inflammatory Myofibroblastic Tumor with Successful Treatment with ALK-Inhibitor
}

\author{
Sanjivini V. Jacob, ${ }^{1}$ John D. Reith, ${ }^{1}$ Angerika Y. Kojima, ${ }^{2}$ William D. Williams, ${ }^{3}$ \\ Chen Liu, ${ }^{1}$ and Lizette Vila Duckworth ${ }^{1}$ \\ ${ }^{1}$ Department of Pathology, Immunology and Laboratory Medicine, University of Florida College of Medicine, P.O. Box 100275, \\ 1600 SW Archer Road, Gainesville, FL 32610-0275, USA \\ ${ }^{2}$ Lake Erie Osteopathic College of Medicine, Bradenton, FL 34211, USA \\ ${ }^{3}$ Department of Pathology, North Broward Health, Pompano Beach, FL 33064, USA
}

Correspondence should be addressed to Lizette Vila Duckworth; lvila@ufl.edu

Received 30 April 2014; Accepted 2 June 2014; Published 18 June 2014

Academic Editor: Zsuzsa Schaff

Copyright (C) 2014 Sanjivini V. Jacob et al. This is an open access article distributed under the Creative Commons Attribution License, which permits unrestricted use, distribution, and reproduction in any medium, provided the original work is properly cited.

\begin{abstract}
Systemic inflammatory myofibroblastic tumor is an exceedingly rare entity. A 45-year-old Hispanic female presented with a 6month history of left-sided thigh pain, low back pain, and generalized weakness. PET/CT scan revealed abnormal activity in the liver, adrenal gland, and pancreas. MRI of the abdomen demonstrated two 6-7 cm masses in the liver. MRI of the lumbar spine demonstrated lesions in the L2 to L4 spinous processes, paraspinal muscles, and subcutaneous tissues, as well as an $8 \mathrm{~mm}$ enhancing intradural lesion at T11, all thought to be metastatic disease. A biopsy of the liver showed portal tract expansion by a spindle cell proliferation rich in inflammation. Tumor cells showed immunoreactivity for smooth muscle actin and anaplastic lymphoma kinase 1(ALK1). Tissue from the L5 vertebra showed a process histologically identical to that seen in the liver. FISH analysis of these lesions demonstrated an ALK (2p23) gene rearrangement. The patient was successfully treated with an ALK-inhibitor, Crizotinib, and is now in complete remission. We present the first reported case, to our knowledge, of inflammatory myofibroblastic tumor with systemic manifestations and ALK translocation. This case is a prime example of how personalized medicine has vastly improved patient care through the use of molecular-targeted therapy.
\end{abstract}

\section{Introduction}

Inflammatory myofibroblastic tumor (IMT) is a mesenchymal neoplasm more commonly occurring during the first two decades of life. IMTs can arise in various anatomic locations [1-6] but occur primarily in the lung [7-9], orbit, retroperitoneum, or abdominopelvic region [10-19]. Lesions may be multifocal. IMT is characterized by a proliferation of myofibroblasts admixed with an inflammatory component of lymphocytes, eosinophils, and plasma cells. Approximately half of IMTs have a rearrangement of the anaplastic lymphoma kinase (ALK) locus on chromosome 2p23, leading to aberrant ALK expression [20, 21]. ALK rearrangements are associated with younger presentation and a more indolent behavior [22]. Local recurrence may occur after surgery with a low risk of distant metastases. A small fraction of IMTs behave more aggressively. The management of IMT can be challenging as there are no established medical treatment protocols [1]. We present the first reported case of systemic IMT with ALK gene rearrangement in a 45-year-old female that responded dramatically to an ALK-inhibitor. This case suggests a therapeutic breakthrough for ALK-positive IMTs using an ALK inhibitor.

\section{Case Report}

A 45-year-old obese Hispanic female, status post cholecystectomy, presented initially with left leg pain, low back pain, generalized weakness, and right-sided blurred vision. Physical examination at that time revealed an abnormal left leg gait and a right lateral strabismus. Laboratory studies revealed mildly elevated liver enzymes (AST 87, ALT 129, total bilirubin 0.9 , and alkaline phosphatase 88 ). Imaging revealed multiple small liver masses and lesions in the lumbar spine. 


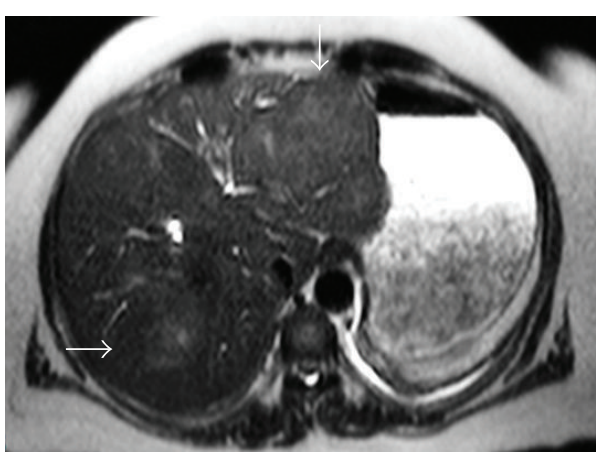

(a)

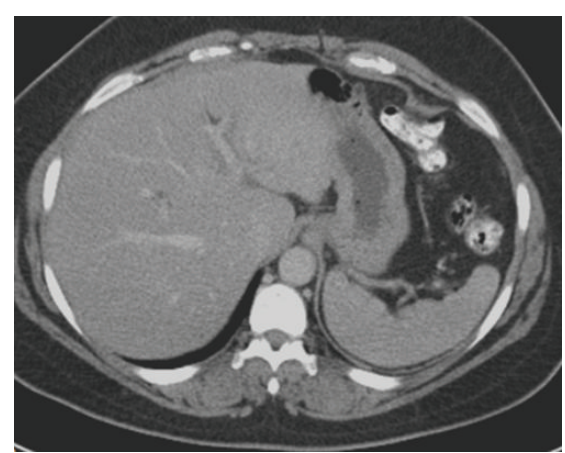

(b)

FIGURE 1: Radiologic appearance of IMT lesions in the liver before (a) and after treatment (b) with ALK-inhibitor. (a) MRI of the abdomen with and without Gadolinium before treatment demonstrates vague poorly defined liver masses in the right and left lobes (arrows) each measuring approximately $6-7 \mathrm{~cm}$. (b) CT of the abdomen obtained at 15-month follow-up demonstrates resolution of the ill-defined liver masses bilaterally following treatment with ALK-inhibitor.

A liver biopsy was performed and was initially interpreted as nonspecific subacute and chronic inflammation with abscess formation. No granulomata, organisms, or evidence of malignancy were seen, and the patient was treated with intravenous antibiotics. Due to high suspicion of tumor in the lumbar region, an exploration of the L3-L4 region was also performed. Operatively, the nerve roots were found to be inflamed in the area, and a proteinaceous cyst was also seen. The patient was subsequently discharged after extensive workup and cultures proved negative.

The patient was readmitted three months after initial discharge due to worsening of low back pain, pain on ambulation, and bilateral lower extremity weakness and numbness, as well as an increase in size of the hepatic and spinal lesions on imaging. Physical exam now showed decreased pinprick, dysesthesias, and weakness with dorsiflexion in the lower extremities bilaterally. Right third cranial nerve palsy was also noted. Repeat MRI of the lumbar spine with and without Gadolinium revealed diffuse enhancing bony lesions involving the L2 to L4 spinous processes, posterior paraspinal muscles, and posterior subcutaneous tissues, and an $8 \mathrm{~mm}$ enhancing intradural lesion at T11, all thought to be metastatic disease. MRI of the abdomen with and without Gadolinium demonstrated a poorly defined $6-7 \mathrm{~cm}$ mass in the left lobe of the liver and a $6 \mathrm{~cm}$ mass in the right lobe of the liver (Figure 1(a)). PET/CT demonstrated abnormal activity in the liver and focal increased activity in the left adrenal gland and head of the pancreas. CA125 was elevated at $88 \mathrm{U} / \mathrm{mL}$ (normal range, less than $35 \mathrm{U} / \mathrm{mL}$ ) but CEA, CA15-3, AFP, and CA19-9 were all within normal range. Serum protein electrophoresis was also performed and was negative for monoclonal gammopathy. The lumbar area was reexplored at that time. Bone and ligamentous material were sent to pathology. A repeat liver biopsy was also performed.

\section{Pathologic Findings}

Histologic examination of the liver and spine lesions revealed a spindle cell proliferation admixed with an inflammatory infiltrate composed of lymphocytes, plasma cells, histiocytes, and eosinophils (Figure 2). The spindle cells were bland in appearance, and no necrosis or atypical mitoses were seen. Immunohistochemical studies revealed that the spindle cells were diffusely immunoreactive for antibodies to smooth muscle actin (SMA), CD68, and exhibited cytoplasmic staining with ALK-1 but were negative for CD117, CD1a, CD23, CD21 (Langerhan cell and dendritic cell markers), EBV, LCA (hematopoetic markers), HepParl (hepatocellular marker), CD34, and S-100 protein. A Giemsa stain was also negative. Subsequently, FISH analysis of both the liver and bone lesions showed an ALK (2p23) gene rearrangement (Figure 3). The patient was given a diagnosis of ALK-rearranged systemic inflammatory myofibroblastic tumor. She subsequently was treated with the ALK-inhibitor Crizotinib and is currently in complete remission (Figure 1(b)) after 27-month follow-up.

\section{Discussion}

Inflammatory myofibroblastic tumor is defined by the World Health Organization as a distinctive lesion of myofibroblastic spindle cells with an inflammatory infiltrate composed of lymphocytes, eosinophils, and plasma cells [23]. It is a rare entity occurring mostly in children and young adults with a mean age of 10 years at diagnosis. Most cases occur within the first 2 decades of life, with a slight female preponderance. These tumors are more commonly found in the soft tissue and viscera of the lungs, mesentery, and abdominopelvic region but can involve any organ system [1-19]. The mainstay of treatment for this tumor is complete surgical excision, although local recurrence and spread have been reported, especially when complete resection is not achieved $[1,10$, $13,15,19,22,24,25]$. Several cases have been treated with chemotherapy, radiation, and/or corticosteroids $[1,3,10,19$, 24]. Systemic or malignant transformation of IMT is rare and has only been reported in a few cases $[19,22,25]$.

Pathologic features of IMTs including tumor size, mitotic rate, presence or absence of necrosis, and cellular atypia do not appear to correlate well with clinical outcome. 


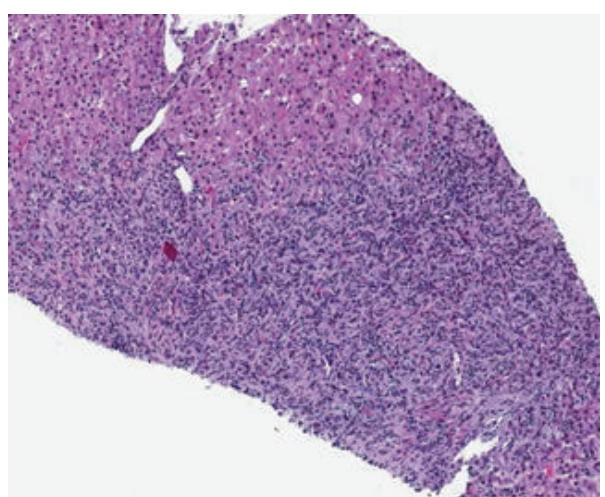

(a)

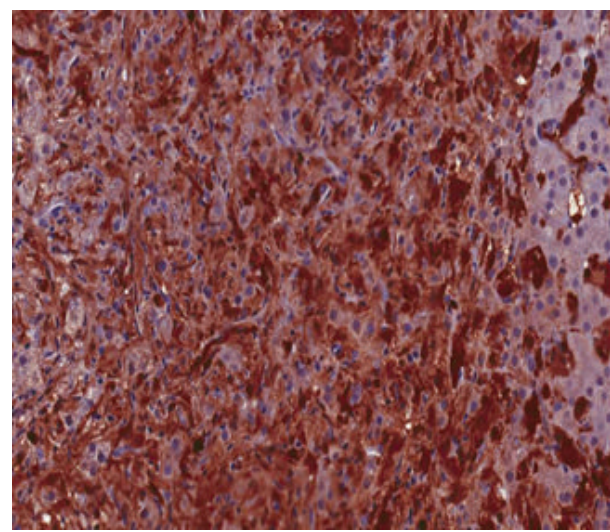

(c)

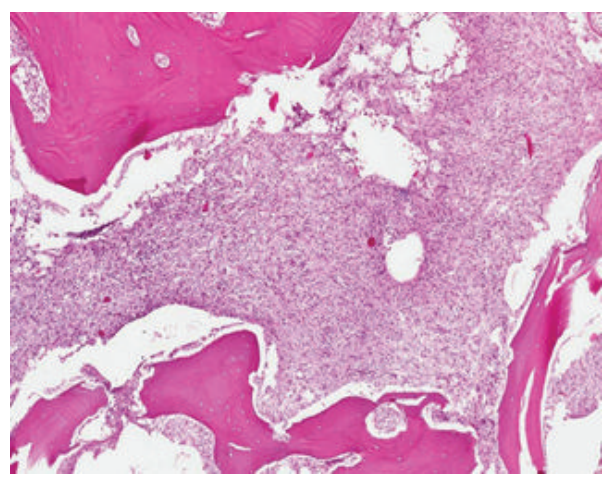

(e)

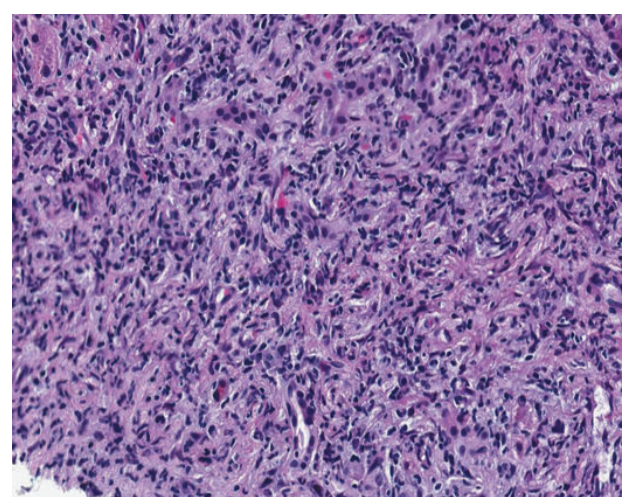

(b)

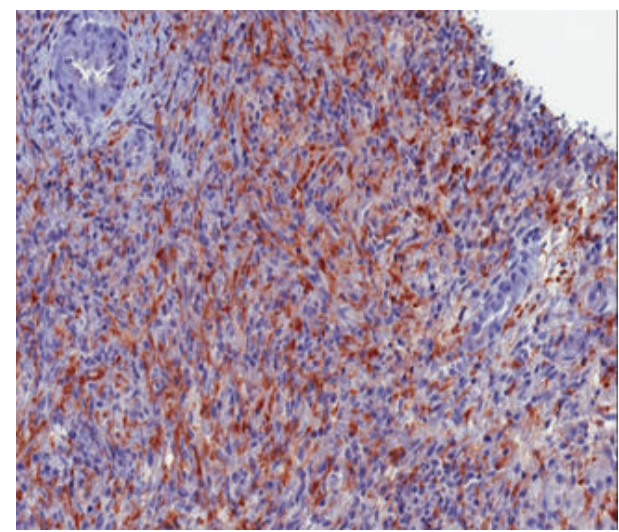

(d)

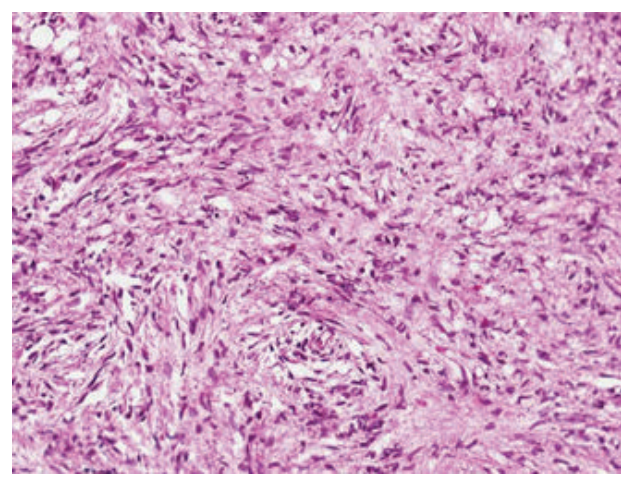

(f)

FIGURE 2: Histologic appearance of IMT lesions in liver and lumbar spine. (a) and (b) Liver tissue with marked inflammatory cells and spindle cell proliferation expanding portal areas and extending into parenchyma. Adjacent normal liver is seen on upper left corner of (a), (H\&E), 5x (a) and 20x (b). (c) Spindle cells show strong and diffuse immunoreactivity to SMA, 20x. (d) Spindle cells are immunoreactive for cytoplasmic anaplastic lymphoma kinase protein (ALK-1), 20x. (e) and (f) Lumbar spine is infiltrated by similar mixture of inflammatory and spindled cells expanding the bone marrow, $\mathrm{H} \& \mathrm{E}, 5 \mathrm{x}$ and $20 \mathrm{x}$.

A small subset of IMTs demonstrate histologic progression to malignancy with increased cellularity/pleomorphism of spindled, round, or polygonal cells with vesicular nuclei, high mitotic rate, and atypical mitoses [26]. However, cases lacking these histologic features have been found to behave in an aggressive manner, and conversely cases with worrisome histologic features have been found to behave indolently [22]. In recent years, a highly aggressive intra-abdominal variant of IMT has been described with epithelioid-to-round cell morphology with nuclear membrane or perinuclear ALK staining and predilection for male patients, which has been termed "epithelioid inflammatory myofibroblastic sarcoma" to convey the malignant nature of this tumor and close relation to IMT [27].

While the nature of IMT is debated and historically thought to be driven by an inflammatory process [22, 27], Griffin et al. demonstrated a chromosomal aberration associated with these tumors [21]. In their study, they found 


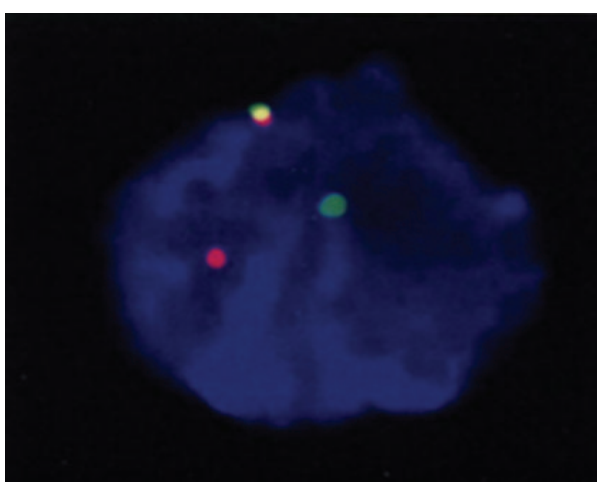

(a)

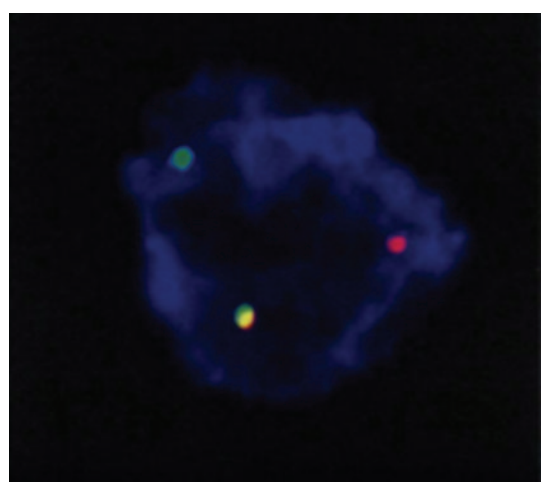

(b)

FIGURE 3: FISH analysis using dual color ALK break apart probe of the liver (a) and lumbar lesions (b) showing abnormal ALK (2p23) gene arrangement (translocation). (a) and (b) This is a "break-apart" probe system, where the fused red/green (overlap as yellow) represents the normal ALK gene locus and the separated red and green signals represent an underlying ALK rearrangement (i.e., translocation). Interphase FISH analysis of at least 200 interphase nuclei was performed with a dual color ALK break probe apart set to detect rearrangement of the ALK locus that is commonly associated with anaplastic large cell lymphoma. The break-apart signal pattern (1R1G1F, one red, one green, and one fusion signal) was observed in $11.5 \%$ of the analyzed nuclei in the liver (a) and in $12.5 \%$ of the analyzed nuclei in the lumbar spine (b). The normal reference is $1.7 \%$, and as such, this represents an abnormal result indicative of ALK gene rearrangement with an unknown chromosomal locus.

that half of IMTs contained a breakage in band p22-24 of chromosome 2, with specific involvement of 2 p23 leading to ALK overexpression [21]. ALK overexpression is typically detectable by immunohistochemistry, which demonstrates cytoplasmic reactivity in IMT, and is also detectable by FISH. Fusion partners of ALK in IMT include TPM3 and TPM4 $[28,29]$, CARS [28, 30], clathrin [31], RAN-BP2 [32], ATIC [33], SEC31L1 [34], and PPF1BP1 [8]. ALK rearrangement has been associated with younger age and local recurrence, but not with distant metastasis [22]. In a study by Coffin et al., 59 cases of IMT were studied, of which 6 were found to have distant metastasis [22]. All six cases with distant metastasis were reported to be ALK negative. To our knowledge, our case represents the first reported case of ALK-translocated systemic IMT in a patient presenting with synchronous tumors in the liver and lumbar spine.

Based on a recent study in the New England Journal of Medicine of successful use of ALK-inhibitor in a treating a patient with ALK-positive IMT [24], our patient was treated with the ALK inhibitor Crizotinib with successful resolution of her lesions and symptoms. After 27-month follow-up, the patient remained in complete clinical and radiologic remission.

In summary, this case represents a distinct presentation of inflammatory myofibroblastic tumor with systemic involvement and ALK gene rearrangement. This case illustrates how personalized medicine through the use of molecular testing has made individual targeted therapy successful.

\section{Conflict of Interests}

The authors declare that there is no conflict of interests regarding the publication of this paper.

\section{References}

[1] N. Gale, N. Zidar, J. Podboj, M. Volavšek, and B. Luzar, "Inflammatory myofibroblastic tumour of paranasal sinuses with fatal outcome: reactive lesion or tumour?" Journal of Clinical Pathology, vol. 56, no. 9, pp. 715-717, 2003.

[2] J. A. Koch, P. Dorn, T. Rausch, H. B. Ris, H. A. Lehr, and S. C. Schäfer, "Inflammatory myofibroblastic tumor of the trachea with concomitant granulomatous lymph node lesions," Case Reports in Medicine, vol. 2011, Article ID 151729, 4 pages, 2011.

[3] A. C. De Oliveira Ribeiro, V. M. Joshi, W. K. Funkhouser, and S. K. Mukherji, "Inflammatory myofibroblastic tumor involving the pterygopalatine fossa," American Journal of Neuroradiology, vol. 22, no. 3, pp. 518-520, 2001.

[4] N. O. Binmadi, H. Packman, J. C. Papadimitriou, and M. Scheper, "Oral inflammatory myofibroblastic tumor: case report and review of literature," The Open Dentistry Journal, vol. 5, no. 1, pp. 66-70, 2011.

[5] R. N. Rao, P. Ranjan, N. Singla, and R. Pandey, "Inflammatory myofibroblastic tumor of the urinary bladder diagnosed by anaplastic lymphoma kinase immunostaining," Urology Annals, vol. 4, no. 2, pp. 115-118, 2012.

[6] S. H. Yoon, K.-J. Kim, S. K. Chung et al., "Inflammatory myofibroblastic tumor in the intradural extramedullary space of the lumbar spine with spondylolisthesis: case report and review of the literature," European Spine Journal, vol. 19, supplement 2, pp. S153-S157, 2010.

[7] C.-K. Chen, C.-I. Jan, J.-S. Tsai et al., "Inflammatory myofibroblastic tumor of the lung- a case report," Journal of Cardiothoracic Surgery, vol. 5, no. 1, article 55, 2010.

[8] K. Takeuchi, M. Soda, Y. Togashi et al., "Pulmonary inflammatory myofibroblastic tumor expressing a novel fusion, PPFIBP1ALK: reappraisal of anti-ALK immunohistochemistry as a tool for novel ALK fusion identification," Clinical Cancer Research, vol. 17, no. 10, pp. 3341-3348, 2011. 
[9] M. Bahadori and A. A. Liebow, "Plasma cell granulomas of the lung," Cancer, vol. 31, no. 1, pp. 191-208, 1973.

[10] O. Firat, S. Ozturk, T. Akalin, and A. Coker, "Inflammatory myofibroblastic tumour," Canadian Journal of Surgery, vol. 52, no. 3, pp. E60-E61, 2009.

[11] T. Plesec, "Gastrointestinal mesenchymal neoplasms other than gastrointestinal stromal tumors: focusing on their molecular aspects," Pathology Research International, vol. 2011, Article ID 952569, 10 pages, 2011.

[12] M. C. Ribeiro, L. R. Lopes, J. C. de Souza Neto, L. R. Meirelles, R. B. de Carvalho, and N. A. Andreollo, "Rare gastric inflammatory myofibroblastic tumor in an adult woman: a case report with review of the literature," Case Reports in Medicine, vol. 2012, Article ID 374070, 4 pages, 2012.

[13] V. L. Pannain, J. V. Passos, A. da Rocha Filho, C. VillelaNogueira, and A. Caroli-Bottino, "Agressive inflammatory myofibroblastic tumor of the liver with underlying schistosomiasis: a case report," World Journal of Gastroenterology, vol. 16, no. 33, pp. 4233-4236, 2010.

[14] E. Y. Kim, I. K. Lee, Y. S. Lee et al., "Inflammatory myofibroblastic tumor in colon," Journal of the Korean Surgical Society, vol. 82, no. 1, pp. 45-49, 2012.

[15] K. Schütte, A. Kandulski, D. Kuester et al., "Inflammatory myofibroblastic tumor of the pancreatic head: an unusual cause of recurrent acute pancreatitis-case presentation of a palliative approach after failed resection and review of the literature," Case Reports in Gastroenterology, vol. 4, no. 3, pp. 443-451, 2010.

[16] B. H. Kye, H. J. Kim, S.-G. Kang, C. Yoo, and H.-M. Cho, "A case of inflammatory myofibroblastic tumor originated from the greater omentum in young adult," Journal of the Korean Surgical Society, vol. 82, no. 6, pp. 380-384, 2012.

[17] B. Cole, H. Zhou, N. McAllister, Z. Afify, and C. M. Coffin, "Inflammatory myofibroblastic tumor with thrombocytosis and a unique chromosomal translocation with ALK rearrangement," Archives of Pathology and Laboratory Medicine, vol. 130, no. 7, pp. 1042-1045, 2006.

[18] W. R. Sukov, J. C. Cheville, A. W. Carlson et al., "Utility of ALK-1 protein expression and ALK rearrangements in distinguishing inflammatory myofibroblastic tumor from malignant spindle cell lesions of the urinary bladder," Modern Pathology, vol. 20, no. 5, pp. 592-603, 2007.

[19] M. K. Dishop, B. W. Warner, L. P. Dehner et al., "Successful treatment of inflammatory myofibroblastic tumor with malignant transformation by surgical resection and chemotherapy," Journal of Pediatric Hematology/Oncology, vol. 25, no. 2, pp. 153158, 2003.

[20] Z. Tothova and A. J. Wagner, "Anaplastic lymphoma kinasedirected therapy in inflammatory myofibroblastic tumors," Current Opinion in Oncology, vol. 24, no. 4, pp. 409-413, 2012.

[21] C. A. Griffin, A. L. Hawkins, C. Dvorak, C. Henkle, T. Ellingham, and E. J. Perlman, "Recurrent involvement of 2p23 in inflammatory myofibroblastic tumors," Cancer Research, vol. 59, no. 12, pp. 2776-2780, 1999.

[22] C. M. Coffin, J. L. Hornick, and C. D. M. Fletcher, "Inflammatory myofibroblastic tumor: comparison of clinicopathologic, histologic, and immunohistochemical features including ALK expression in atypical and aggressive cases," American Journal of Surgical Pathology, vol. 31, no. 4, pp. 509-520, 2007.

[23] C. D. M. Fletcher, K. K. Unni, and F. Mertens, Eds., World Health Organization Classification of Tumours. Pathology and Genetics of Tumours of Soft Tissue and Bone, IARC Press, Lyon, France, 2002.
[24] J. E. Butrynski, D. R. D'Adamo, J. L. Hornick et al., "Crizotinib in ALK-rearranged inflammatory myofibroblastic tumor," The New England Journal of Medicine, vol. 363, no. 18, pp. 1727-1733, 2010.

[25] H.-D. Zhao, T. Wu, J.-Q. Wang et al., "Primary inflammatory myofibroblastic tumor of the breast with rapid recurrence and metastasis: a case report," Oncology Letters, vol. 5, no. 1, pp. 97100, 2012.

[26] L. R. Donner, R. A. Trompler, and R. R. White IV, "Progression of inflammatory myofibroblastic tumor (inflammatory pseudotumor) or soft tissue into sarcoma after several recurrences," Human Pathology, vol. 27, no. 10, pp. 1095-1098, 1996.

[27] A. Mariño-Enríquez, W.-L. Wang, A. Roy et al., "Epithelioid inflammatory myofibroblastic sarcoma: an aggressive intraabdominal variant of inflammatory myofibroblastic tumor with nuclear membrane or perinuclear alk," American Journal of Surgical Pathology, vol. 35, no. 1, pp. 135-144, 2011.

[28] J. Cools, I. Wlodarska, R. Somers et al., "Identification of novel fusion partners of ALK, the anaplastic lymphoma kinase, in anaplastic large-cell lymphoma and inflammatory myofibroblastic tumor," Genes Chromosomes and Cancer, vol. 34, no. 4, pp. 354-362, 2002.

[29] B. Lawrence, A. Perez-Atayde, M. K. Hibbard et al., “TPM3ALK and TPM4-ALK oncogenes in inflammatory myofibroblastic tumors," American Journal of Pathology, vol. 157, no. 2, pp. 377-384, 2000.

[30] L. V. Debelenko, D. C. Arthur, S. D. Pack, L. J. Helman, D. S. Schrump, and M. Tsokos, "Identification of CARS-ALK fusion in primary and metastatic lesions of an inflammatory myofibroblastic tumor," Laboratory Investigation, vol. 83, no. 9, pp. 1255-1265, 2003.

[31] J. A. Bridge, M. Kanamori, Z. Ma et al., "Fusion of the ALK gene to the clathrin heavy chain gene, CLTC, in inflammatory myofibroblastic tumor," American Journal of Pathology, vol. 159, no. 2, pp. 411-415, 2001.

[32] Z. Ma, D. A. Hill, M. H. Collins et al., "Fusion of ALK to the Ran-binding protein 2 (RANBP2) gene in inflammatory myofibroblastic tumor," Genes Chromosomes and Cancer, vol. 37, no. 1, pp. 98-105, 2003.

[33] M. Debiec-Rychter, P. Marynen, A. Hagemeijer, and P. Pauwels, "ALK-ATIC fusion in urinary bladder inflammatory myofibroblastic tumor," Genes Chromosomes and Cancer, vol. 38, no. 2, pp. 187-190, 2003.

[34] I. Panagopoulos, T. Nilsson, H. A. Domanski et al., "Fusion of the SEC31L1 and ALK genes in an inflammatory myofibroblastic tumor," International Journal of Cancer, vol. 118, no. 5, pp. 11811186, 2006. 


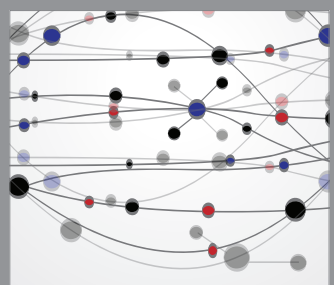

The Scientific World Journal
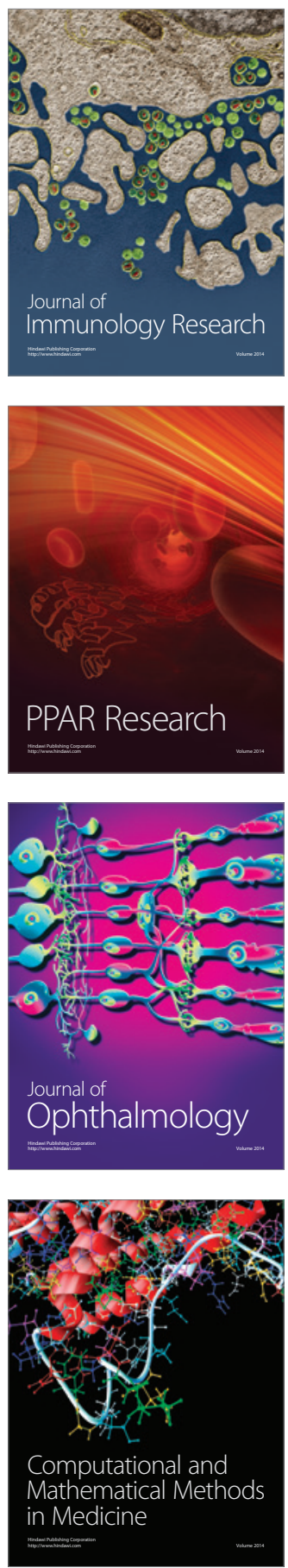

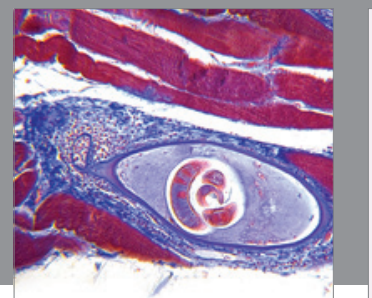

Gastroenterology

Research and Practice
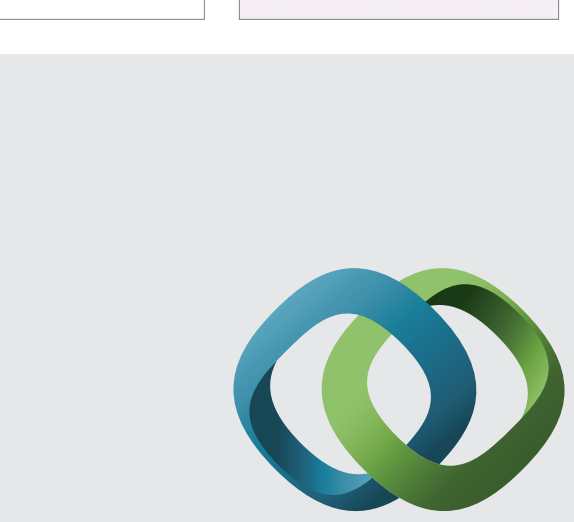

\section{Hindawi}

Submit your manuscripts at

http://www.hindawi.com
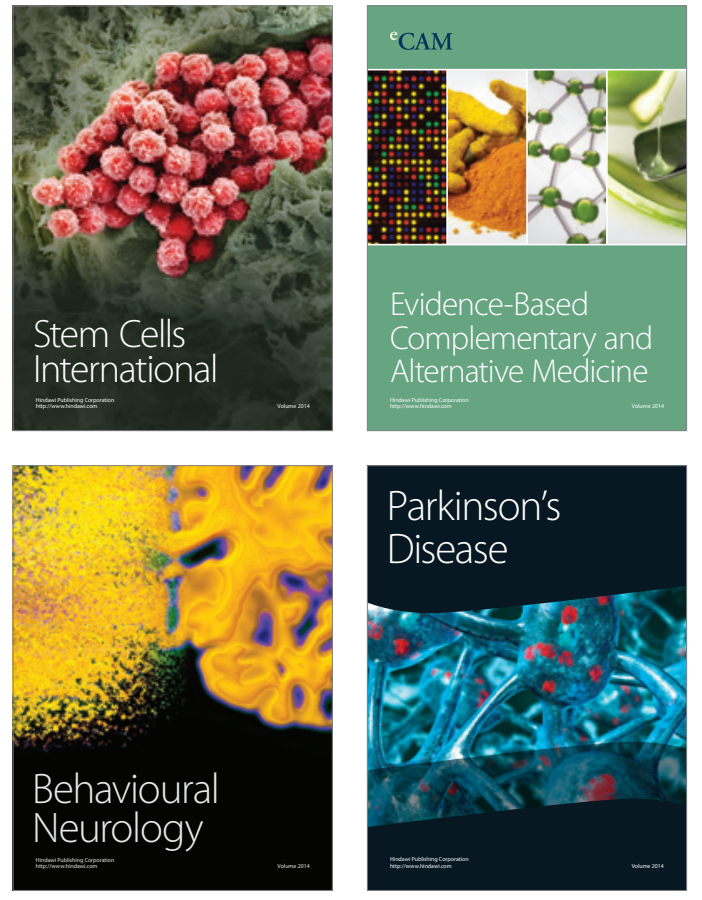
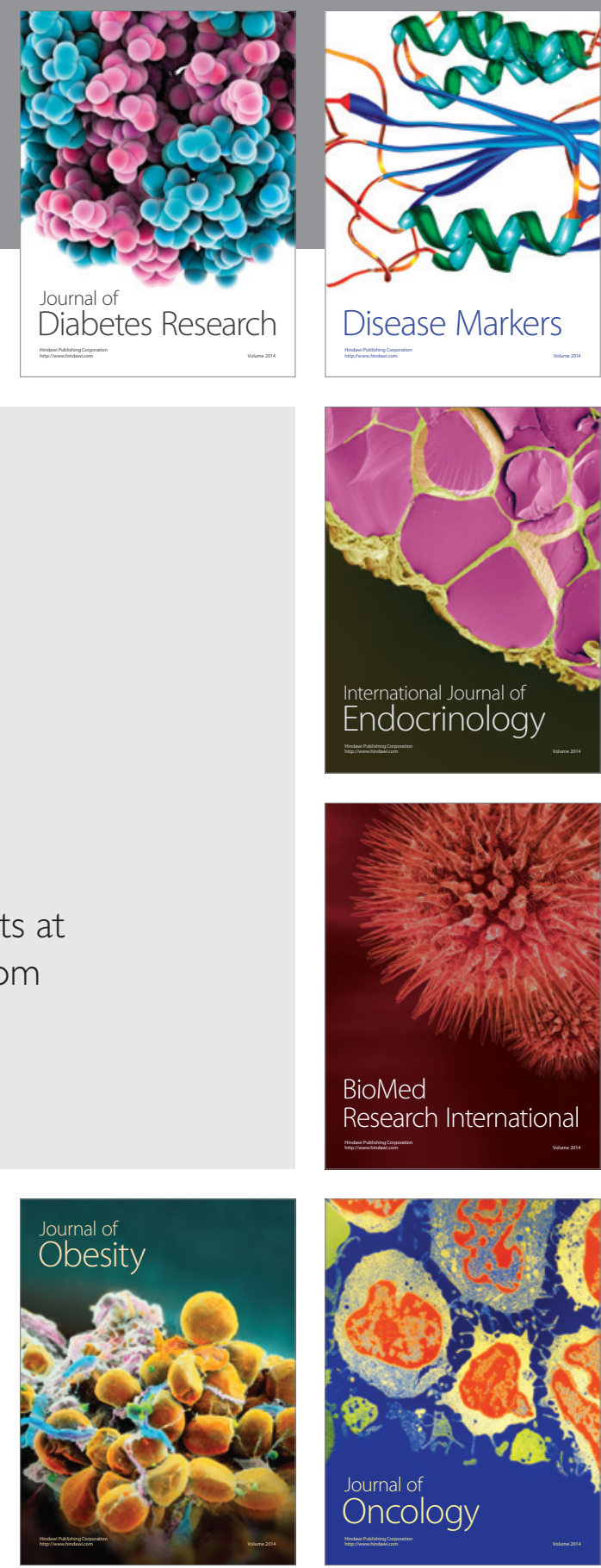

Disease Markers
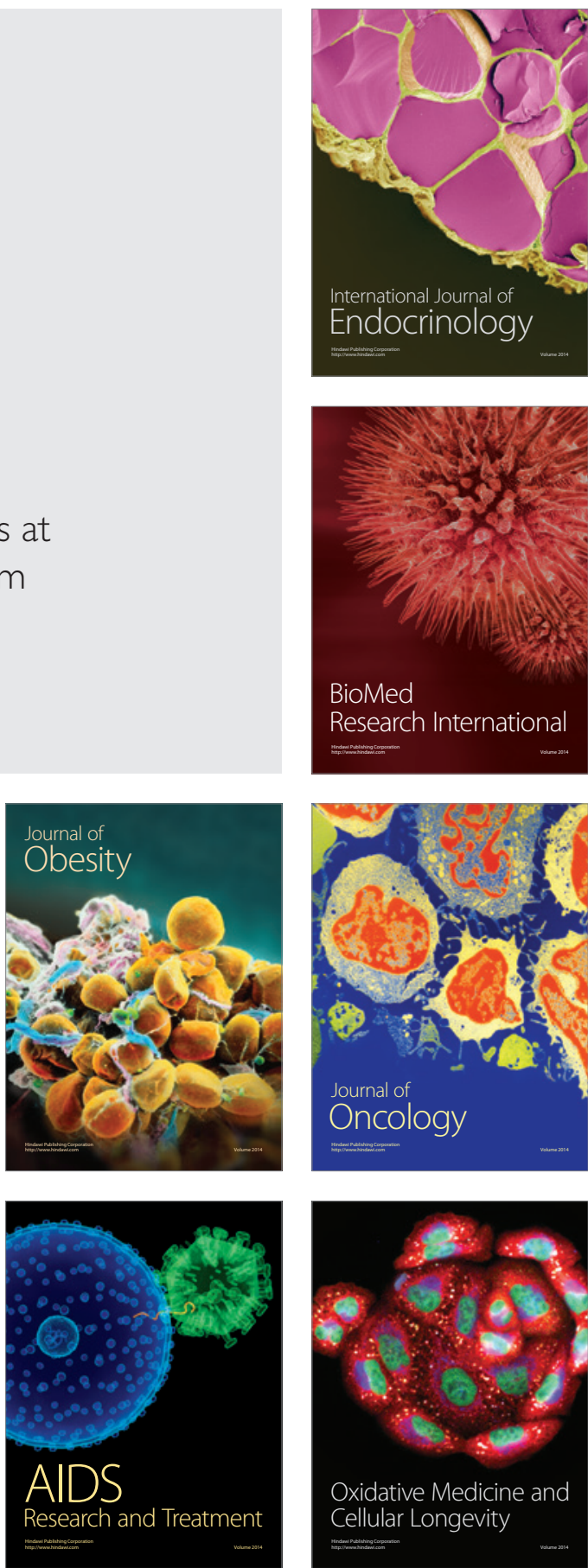